\title{
Les mécanismes du Naturalisme à l'épreuve : la télégonie dans Madeleine Férat
}

\author{
María RodRíGUEz ÁlVAREZ \\ Universidad de Oviedo \\ ralvarezmaria@uniovi.es \\ ORCID : 0000-0002-9062-1470
}

\section{Resumen}

Una de las claves del éxito literario de Émile Zola reside en la aplicación de los avances científicos del siglo XIX a la ficción narrativa de una forma tan natural como sólo alguien con un conocimiento profundo de la crítica y la literatura sabe hacer. Dicha perfección estilística es producto de un proceso de ensayo y error que culmina con Les Rougon Macquart pero que tiene en Madeleine Férat y su aproximación a la telegonía uno de sus episodios más destacados, pero también más desconocidos. Esta teoría reproductiva es a la vez un pretexto para diseccionar milimétricamente la sociedad burguesa y el punto de partida de la fecunda relación entre ciencia y literatura tan característica del Naturalismo.

Palabras clave: Émile Zola, Teoría de la impregnación, Epistemocrítica, Herencia, Naturalización.

\section{Résumé}

L'une des clés du succès littéraire de Zola réside dans l'application des résultats du progrès scientifique du $\mathrm{XIX}^{\mathrm{e}}$ siècle dans la fiction romanesque d'une façon si naturelle dont seul un maître aussi bien de la critique que de la littérature pourrait être responsable. Cette perfection dans le style est le produit d'un processus d'essai et erreur qui aboutit dans Les Rougon Macquart, mais dont Madeleine Férat et son approche à la télégonie constituent un épisode majeur et pourtant très inconnu. Cette théorie reproductive est, à la fois, un prétexte pour analyser en profondeur la société bourgeoise et le point de départ du rapport fécond entre science et littérature qui caractérise le Naturalisme.

Mots clé : Émile Zola, Théorie de l'imprégnation, Épistémocritique, Hérédité, Naturalisation.

\begin{abstract}
One of the keys of Zola's literary success relies on the integration of scientific developments from the 19th century into the narrative texts in such a natural way that
\end{abstract}

* Artículo recibido el 25/02/2020, aceptado el 25/10/2020. 
only someone with a deep knowledge of both criticism and literature would be able to. This stylistic perfection is the result of a trial and error process that culminates in Les Rougon Macquart but in which Madeleine Férat and its approach to telegony play an outstanding but unknown role. This reproductive theory is at the same time an excuse to undergo a deep analysis of the bourgeoisie but also the beginning of the productive relationship between science and literature, a major feature in Naturalism.

Keywords: Émile Zola, Theory of impregnation, Epistemocriticism, Heredity, Naturalisation.

\section{Introduction}

L'image d'Émile Zola comme fondateur d'une école dont la conception moderne reposait sur l'interaction de la science et de la littérature demeure encore de nos jours connue de tous. Bien que relevant en partie du poncif, il ne semble pas possible néanmoins d'offrir un portrait fidèle du chef de file du Naturalisme sans tenir compte des idées qu'il avait prônées à ce sujet dans ses essais et appliquées dans ses œuvres de fiction.

Au moment où l'industrialisation a attelé l'homme à une machine, Zola a entrepris sa propre révolution en nouant des rapports forts entre des notions telles que science et littérature, entre écriture et critique, qui étaient jusque là nettement indépendantes et présentant des caractéristiques bien différenciées. Une tâche complexe et avec beaucoup d'implications, non seulement du point de vue intellectuel, mais aussi social, car la performance technique avait anéanti l'ordre traditionnel et noyé le monde dans le positivisme et l'espoir dans le progrès. Zola s'empare vite de cet esprit de l'époque. Dans une explication plus en profondeur du but de son œuvre théorique, il revendique que l'application de la science à tous les domaines de la vie pousse celle-ci hors de ses limites, encore très floues. Plutôt que le produit d'un intérêt personnel, son but littéraire est enraciné dans la réalité sociale de l'époque, qui assistait à l'éclatement des disciplines scientifiques et à la fondation de la science en tant que telle, à un stade très embryonnaire et en suivant des pas encore très dubitatifs.

Il n'est donc pas étonnant que Zola, qui était par ailleurs fils d'un ingénieur, ait trouvé dans l'interaction entre science et littérature la formule de la réussite littéraire et théorique, dont les expressions les plus remarquables à ce propos sont Les Rougon Macquart (1871-1893) et Le Roman expérimental (1880). Dans ce recueil d'articles, Zola (2006 : 121) exprime son objectif au moyen d'une phrase qui demeure encore célèbre: «le naturalisme [est] la formule de la science moderne appliquée à la littérature». Chef de file de ce courant littéraire malgré lui, Zola, grand admirateur de Balzac, avoue très tôt son intention d'entreprendre sa Comédie Humaine dans la plus grande rigueur scientifique. Avec la série des Rougon, il réussit à montrer l'homme tel qu'il est, non pas un être libre et autonome, mais plutôt le produit de multiples phénomènes et causes. Une telle perspective, alors nouvelle, ouvrait un chemin pour trouver des solutions « scienti- 
fiques » aux problèmes sociaux, ainsi que le suggère déjà le sous titre de la collection : "Histoire naturelle et sociale d'une famille sous le Second Empire ».

À travers ce projet, Zola parviendra à fondre l'écrivain et le théoricien qu'il était, en trouvant l'inspiration chez des auteurs de référence aussi bien dans le domaine de la science que de la critique. Parmi les premiers, on signalera Hippolyte Taine (1828-1893) et son œuvre Philosophie de l'art, que Zola reconnaissait dans Le Roman expérimental comme le responsable de l'accès de la critique littéraire à la modernité. En effet, ce philosophe, critique et historien considérait que la théorie était, comme le monde physique, soumise à des lois qu'il fallait énoncer au moyen de l'analyse et la pure observation (Mourad, 2007). Parmi les influences plus spécifiquement scientifiques, on ne manquera pas de mentionner ici le Docteur Lucas (1808-1885) et son Traité de l'hérédité naturelle ainsi que Charles Darwin (1809-1882), qui avait bouleversé le monde des sciences naturelles en 1859 avec la publication de L'origine des espèces où il exposait sa théorie de la sélection naturelle, texte fondateur de la théorie de l'évolution. Mais le savant qui a exercé la plus grande influence sur Émile Zola n'est autre que Claude Bernard (1813-1878). Fondateur de la physiologie, son Introduction à l'étude de la médecine expérimentale a représenté pour lui une référence incontournable pour l'écriture de ses romans, dont les traces restent bien visibles dès le titre dans Le Roman expérimental.

\section{Claude Bernard et les origines du savant}

On conviendra que l'étude de la genèse, et surtout des conséquences du progrès exorbitant vécu au $\mathrm{XIX}^{\mathrm{e}}$ siècle sous le règne tyrannique de la Révolution Industrielle, néglige assez fréquemment les disciplines non techniques. L'époque représente pourtant l'entrée dans une nouvelle ère pour les sciences biomédicales, grâce notamment au progrès scientifique généralisé, mais surtout au développement de la physique et de la chimie. Ces disciplines ont joué dès lors un grand rôle dans l'élargissement des connaissances au sein du paradigme scientifique, mais aussi dans le surgissement de nouvelles disciplines, telles que la biochimie ou, au $\mathrm{XX}^{\mathrm{e}}$ siècle, la biologie moléculaire. Des découvertes comme la structure de la molécule de l'ADN ou le séquençage du génome humain auraient été impossibles sans les contributions de la chimie, mais aussi sans l'une des premières disciplines pour lesquelles elle a constitué une sorte d'échafaudage: la physiologie. Certes elle existait au XIX ${ }^{\mathrm{e}}$ siècle, mais elle était associée à l'anatomie. Les travaux précurseurs de François Magendie dans ce domaine pendant la première moitié de ce siècle ont démontré le besoin d'une prise de chemins indépendants.

Les travaux de Claude Bernard, disciple de Magendie, sur l'appareil digestif et surtout sur le foie - il a découvert sa fonction de producteur de matière sucrée pour le système circulatoire - l'ont consacré comme le grand initiateur de la physiologie et lui ont ouvert la porte des institutions les plus prestigieuses de la France de l'époque : l'Académie des Sciences en 1854 et le Collège de France, dont il a fini par être le directeur. S'il était déjà considéré à son époque comme un référent pour le développement des sciences, ce n'était pas uniquement grâce aux 
résultats de ses recherches, mais bien par l'importance et la rigueur dans sa présentation, ce qui fait de lui le savant qui a posé les bases de la méthode scientifique et, par conséquent, de cette profession.

Les processus d'expérimentation jouent un rôle prépondérant dans le développement de la physiologie, comme l'a rendu manifeste le devenir même de celle-ci. À une époque où la science n'a pu connaître un essor plus rapide, faute de moyens, Claude Bernard cultivait déjà la technique de la vivisection, c'est-à-dire, la dissection faite sur un animal vivant, ce qui permettait d'observer les processus biologiques in situ, un monde plein de possibilités pour la physiologie. Claude Bernard (1872 : 209) a fait du laboratoire son habitat pour dépasser les propres limitations qu'il avoue avoir éprouvées par manque de toute infrastructure scientifique : "J'ai connu la douleur du savant qui, faute de moyens matériels, ne peut entreprendre ou réaliser des expériences qu'il conçoit, et est obligé de renoncer à certaines recherches, ou de laisser sa découverte à l'état d'ébauche ». La publication de l'Introduction à l'étude de la médecine expérimentale en 1865 signifie l'abandon de ses craintes, l'exemplification de la rigueur scientifique, l'explicitation de l'interdépendance entre physiologie, pathologie et thérapeutique, ainsi que la fixation des règles de ce qui sera par la suite la médecine moderne. Cette œuvre, qui n'allait être que la préface de Principes de médicine expérimentale, ouvrage finalement inachevé, devient un événement majeur pour l'avenir des sciences naturelles qui, dès lors, ont connu une grande transformation.

Le travail simultané de la physique, de la chimie et de la médecine entre les années 1860 et 1890, qui a contribué de façon déterminante au dépassement progressif des difficultés inhérentes au statut scientifique à l'époque, a été décisif pour le développement d'autres disciplines comme la génétique. Cette branche de la biologie, qui prit son véritable essor avec le nouveau siècle lors de la diffusion des travaux de Georges Mendel (1822-1884), est fondamentale pour comprendre l'œuvre littéraire d'Émile Zola dans sa juste mesure.

La construction de la génétique moderne peut, en quelque sorte, ressembler aux étapes de la méthode scientifique, ou même à la construction d'un roman, d'autant qu'elle s'est produite comme s'il s'agissait de la remise dans l'ordre des pièces d'un puzzle. Entre 1870 et 1880, la situation est celle que l'on pourrait considérer «normative » dans un horizon de rupture comme celui du dernier tiers du $\mathrm{XIX}^{\mathrm{e}}$ siècle pour la science, où des théories reproductives et de l'hérédité variées, valables et erronées, cohabitaient dans un même paradigme. La situation pourrait avoir été radicalement différente si le résultat des travaux de Mendel, publiés originalement en 1865, avaient eu quelque retentissement à l'époque. On a dû cependant attendre jusqu'au début du $\mathrm{XX}^{\mathrm{e}}$ siècle pour que les postulats de Mendel soient acceptés par les académiciens, ce qui a supposé un véritable point de départ pour le développement de la génétique moderne. Sans doute, une acceptation et une diffusion moins tardive de ces idées auraient rendu plus facile la compréhension et peut-être l'accueil favorable des hypothèses de Darwin car, pour étonnant que cela puisse paraître aujourd'hui, ses postulats sur l'évolution sont nés sans 
une théorie de l'hérédité qui les soutienne, vu que « les scientifiques [à l'époque] se consacraient plutôt à des hypothèses fondées sur des aspects de biologie générale, à l'histoire anecdotique ou au travail expérimental mené par les agriculteurs et les éleveurs ${ }^{1} \gg$ (Carlson, $\left.1985: 774\right)$.

La divulgation de la théorie mendélienne a contribué à fermer le cercle darwinien qui s'était déjà enrichi par les progrès atteints par August Weismann, biologiste et théoricien de l'évolution, dont les travaux ont été consacrés à la transmission des caractères héréditaires. Ce savant est parvenu à distinguer l'existence d'une substance responsable de celle-ci, qu'il a appelée «plasma germinatif », dans les cellules germinales - reproductives -, qu'il distinguait déjà des somatiques, c'est-à-dire, non-reproductives. Cette théorie a devancé les études sur l'importance du noyau cellulaire pour les processus de reproduction qui ont commencé à se clarifier entre les années 1870-1880, et qui ont mené à l'identification des chromosomes et à la description de la distribution uniforme de ceux d'origine maternelle et paternelle lors de la division cellulaire (Sánchez Ron, 2001).

Le puzzle de la génétique moderne s'est beaucoup perfectionné tout au long du $\mathrm{XX}^{\mathrm{e}}$ siècle par le travail interdisciplinaire, ce qui a donné naissance à des branches comme la biotechnologie et l'ingénierie génétique, mais surtout grâce à l'étude du rôle que joue l'ambiance dans les processus de l'hérédité. Le développement scientifique dans le domaine de l'hérédité (génétique, physiologie, biochimie...) a été si important que, en un peu moins d'un siècle, on est passé d'ignorer l'existence même du matériel génétique à identifier les différences épigénétiques, c'est-à-dire, à remarquer l'influence des agents externes, directement liés aux conditions de vie et à la distribution géographique de la population sur la manifestation de quelques gènes. On parvient ainsi à décrire des facteurs comme la nourriture, le sommeil, le sport ou le stress, comme étant capables de modifier l'expression de quelques gènes, susceptibles d'être transmis aux descendants.

Il existait au XIX ${ }^{\mathrm{e}}$ siècle une théorie qui serait de nos jours considérée peu scientifique et de nature intuitive, en fait il s'agirait plutôt d'une doctrine, qui défendait l'influence de l'ambiance sur l'héritage de caractères et que l'on connaissait sous le nom de «l'imprégnation par le regard ». Il s'agissait de «la croyance selon laquelle un enfant pouvait ressembler à une personne ou une chose qui aurait impressionné sa mère lors des rapports sexuels ou lors de la gestation » (Wilgaux, 2006 : 345). Pour saugrenue qu'elle puisse paraître, cette idée a réussi à être acceptée au sein des théories scientifiques pendant plus d'un demi siècle (18601920), dans le contexte d'un paradigme scientifique plus large - aujourd'hui considéré plutôt une mythologie -, celui de la télégonie, dans laquelle s'inscrivent plusieurs variantes des théories dites de l'imprégnation, dont celle « d'imprégnation par le regard». Avant d'entrer dans des considérations de rigueur scientifique ou de nuances chronologiques au sein de la propre théorie selon

\footnotetext{
${ }^{1}$ C'est nous qui traduisons à partir du texte suivant: « scientists were content to theorize about it on the bases of more general biological concerns, anecdotal history, or the limited range of experimental work carried out by horticulturalists and animal breeders ».
} 
sa conception originaire du $\mathrm{XIX}^{\mathrm{e}}$ siècle, on prendra la définition de télégonie d'Anne Carol (2006 : 11) comme point de départ : «La télégonie est la croyance selon laquelle une femme, fécondée ou déflorée par un premier homme, produit ensuite des enfants à sa ressemblance quel que soit le géniteur ». Tel est le sujet auquel seront consacrées les pages qui vont suivre et l'axe thématique que Zola a choisi pour le dernier de ses romans avant la série de Les Rougon Macquart: Madeleine Férat ${ }^{2}$.

Les définitions du phénomène à l'époque ne sont pas très différentes de celle que Carol présente aujourd'hui, comme le révèle l'approche faite par plusieurs auteurs tout au long de la deuxième moitié du XIX ${ }^{\mathrm{e}}$ siècle. Prenons celle de Henry de Varigny présentée dans le Journal des débats politiques et littéraires du 9 septembre 1897: «Une forme d'hérédité particulière qui consiste en ceci qu'une mère peut transmettre à la progéniture d'un second lit des caractères appartenant au père de la progéniture d'un premier lit ».

Quand on consulte d'autres documents plus ou moins contemporains à celui de Varigny, on constate que celui-ci suit une ligne de continuité par rapport au concept de télégonie fixée pendant sa période d'essor, comprise, d'après Carol, entre 1860 et 1920 . Ce même article sert pourtant également à constater les divergences dans la terminologie employée. À la dite «télégonie », terme qui date en réalité du début du $\mathrm{XX}^{\mathrm{e}}$ siècle, ou «mesaillance initiale », qui reçoivent un certain consensus (Lucas-Championnière, 1915: 458), il faut ajouter celui d' «imprégnation », souvent employé comme synonyme de «télégonie », plutôt par un processus métonymique que par une équivalence scientifiquement fondée. Raoul Anthony (1900 : 29) éclaircissait cet emploi erroné, mais pourtant assez fréquent, ainsi:

Le terme télégonie constate simplement un fait, l'apparition chez des produits ultérieurs de caractères appartenant à un premier procréateur, mais ne cherche nullement à l'expliquer; le terme imprégnation au contraire désigne une modification des parties somatiques de la mère (organisme entier ou organes génitaux internes seulement) sous l'influence d'un fotus qu'elle porte ou peut-être même simplement, suivant certains auteurs, du liquide fécondateur; le mot imprégnation ne veut dire rien de plus et ne doit donc pas être considéré comme le synonyme de télégonie. L'imprégnation, est pour ceux qui admettent que cette influence se transmet dans la suite de la mère à des foetus ultérieurs, la condition de la télégonie; pour ceux au contraire qui admettent la possibilité d'une survivance plus ou moins

\footnotetext{
${ }^{2}$ Madeleine Férat est l'un des romans moins célèbres de Zola. Paru en 1868, il sera réédité en 1976 dans Le livre de Poche, mais il faudra attendre 1999 pour voir apparaître une nouvelle édition, celle de Henri Mitterand chez « Mémoire du livre », dont nous nous servons justement ici. Depuis le roman était devenu introuvable. Heureusement, cet été dernier, la maison «Le Chat Rouge» a lancé une nouvelle édition avec une préface de Gérald Duchemin où l'on présente le livre comme une perle rare.
} 
longue des spermatozoïdes dans les voies génitales de la mère, l'imprégnation n'est pas nécessaire pour expliquer la télégonie. Le mot télégonie constate un fait; le mot imprégnation vise à une explication théorique; il en est de même d'ailleurs du terme hérédité fraternelle que l'on emploie parfois. C'est pour cette raison que le terme télégonie quoique plus obscur semble préférable à tous les autres.

Avant de poursuivre notre analyse, il faut faire une considération sur l'emploi de cette terminologie dans Madeleine Férat. On verra que la distinction entre fait et modification physiologique reçue dans la citation d'Anthony n'est pas suivie par Zola dans le roman, qui utilise ces deux notions presque indistinctement et peut-être de façon parfois erronée. De manière générale, on préférera ici plutôt le mot «télégonie » pour essayer d'être rigoureux avec les situations présentées, mais il faut avertir le lecteur que l'approche du romancier à la question nous conduira parfois à employer le terme « imprégnation ».

Le début de la citation antérieure laisse entrevoir quelques tendances qui cohabitent au sein de l'imprégnation pour expliquer les mécanismes de celle-ci. De façon générale, on nous en présente deux qui s'organisent selon que l'on se situe dans la perspective de la femme ou bien dans celle de l'homme. Le premier cas tient un rapport étroit avec la théorie de «l'imprégnation par le regard » et avec bien d'autres semblables, comme celle des effets de l'imagination sur la mère, assez prolifique dans le monde littéraire avant que la télégonie ne soit devenue matériel mythique une fois les postulats de Mendel acceptés. Pourtant, il semble que la tradition épistémologique de l'imprégnation commence déjà avec Aristote, qui constatait un rôle prééminent de l'homme dans la fécondation au détriment de la passivité de la femme (Olrik, 1986). Sa grande période d'essor se concentre sur la deuxième moitié du $\mathrm{XIX}^{\mathrm{e}}$ siècle, avec l'œuvre de Zola, bien évidemment, mais aussi grâce à sa présence dans quelques œuvres des auteurs scandinaves August Strindberg et, surtout, Henrik Ibsen. À ce propos, Carlsen (1985 : 774) considère que «l'hérédité donnait une source commune de métaphore, permettant ainsi à Ibsen d'exprimer sa fascination envers l'influence du passé dans le présent et Strindberg de s'occuper du rapport homme-femme et de la relation problématique entre les géniteurs et leur descendance $»^{3}$.

Quant à la notion spécifique de l'imprégnation par le regard, on la trouve, par exemple, à la base de l'un des romans les plus représentatifs du XVIII ${ }^{\mathrm{e}}$ siècle anglais, Vie et opinons de Tristam Shandy, gentilhomme de Laurence Sterne, publié en 1749, plus d'un siècle avant Madeleine Férat. Le héros du roman attribut les maux de son existence aux circonstances de sa procréation, car lors du coït qui l'avait engendré, sa mère avait osé demander à son père:

\footnotetext{
${ }^{3}$ C'est nous qui traduisons à partir du texte suivant: « Heredity provided a common source of metaphor, helping Ibsen to express his fascination with the influence of the past on the present and Strindberg to deal with male-female relationships and with the problematic relation of fathers to their offspring ».
} 
Dites-moi, mon cher, demanda ma mère, n'avez-vous pas oublié de monter la pendule ?---------- Bon Dieu! s'écria mon père, qui, tout en faisant cette exclamation, prit soin de modérer sa voix, ----------- jamais femme, depuis la création du monde, a-t-elle interrompu un homme par une si sotte question ? $(1749: 2)$.

Chez Sterne, bien qu'avec une ironie visible, les circonstances dans lesquelles se produit l'acte de procréation étaient déterminantes pour la destinée de l'enfant. De telles théories étaient donc assez populaires et même acceptées par des savants de premier ordre. Zola aurait pu en avoir connaissance à travers Claude Bernard, car, même si largement débattue à l'époque, une troisième explication des mécanismes de l'imprégnation s'est faite une place au sein de l'œuvre de quelques scientifiques très réputés, parmi lesquels Bernard et même Charles Darwin. Les deux partageaient une vision de l' «imprégnation imparfaite » des ovaires lors du premier coït de la femme avec un homme et qui serait complétée, selon la théorie bernardienne, par un «stimulus extérieur » pour mûrir le matériel préexistant. De façon technique, cette imprégnation imparfaite, un cas de déformation de la reproduction sexuelle par ovule non-fécondée, a reçu le nom de parthénogénèse.

Ces idées s'inscrivent dans la ligne du vitalisme, qui défend l'existence d'une force inhérente aux êtres vivants qui les distingue des inanimés. Le vitalisme a été le champ de bataille, qui, vers le tournant du siècle, s'est ouvert entre ceux qui étaient pour ou contre la « doctrine » de la télégonie, d'après la nomenclature employée dans le Journal de médecine et de chirurgie pratiques à l'usage des médecins praticiens du 15 juin 1915. La question était assez en vogue à l'époque en France, surtout pendant et même après la Grande Guerre : en effet, un nombre important de femmes qui avaient eu un enfant de leur violeur allemand refusèrent par la suite de tomber à nouveau enceinte de peur que le nouveau né n'ait les traits du soldat étranger.Sans doute la constatation d'un manque de ressemblance entre ce dernier et l'enfant a finalement contribué à discréditer une telle théorie, dont les premiers constats avaient été faits par des éleveurs, notamment dans le contexte de la zootechnie.

Les observations que Lord Morton avaient faites pendant la décennie de 1820 sur une jument arabe fécondée pour la première fois par un couagga, un type de zèbre d'Afrique du Sud, ont été à ce propos une référence récurrente vers la moitié du XIX ${ }^{\mathrm{e}}$ siècle. Selon lui, la descendance avait été un hybride avec des traits de ce dernier. Fécondée à nouveau quelques années après par un cheval arabe, la jument avait donné naissance à une descendance à traits arabes mais avec des zébrures au pelage. Une observation analogue faite avec la descendance d'une chatte de race «anoure » de l'Île de Man, sans rapport antérieur aux chats communs, et fécondée successivement par ceux-ci, avait également démontré la pertinence de l'imprégnation. La descendance présentait aussi une sorte de mosaïque dans les traits extérieurs, connue comme gynandromorphisme, dont le terme date déjà de 1730. 
Malgré l'apparente scientificité que l'appui bernardien et darwinien semble apporter à la théorie, la réalité est que, déjà à l'époque, les positions au sein du monde scientifique par rapport à la télégonie étaient bien divisées. On peut classer les critiques sur des aspects bien nets et qui entrent directement en conflit avec les postulats de Claude Bernard sur la méthode expérimentale : le manque de systématisation des observations dans le monde animal et sa difficile adaptation pour trouver une explication validée sur le plan de la reproduction humaine. Malgré les expériences auparavant citées, ce manque qui a accompagné toute tentative d'étude de la télégonie fait de n'importe quel cas de celle-ci un événement exceptionnel, dont les observations, sans le moindre résultat solide, manquent de toute rigueur scientifique (Varigny, 1897). Le rejet de la théorie du coït ultérieur par certains secteurs, liée à des phénomènes de superfétation, n'a pas mis cependant fin au débat sur la télégonie. La quête d'une cause valable pouvant être considérée dans le contexte de la pleine expansion des sciences naturelles a nourri la discussion sur l'atavisme.

Cette idée constitue un sujet commun dans les discussions sur la télégonie qui ont eu lieu à ce propos. Prenons comme exemple la communication d'Anthony le 4 mai 1899 à la Société d'Anthropologie de Paris sur la descendance de la chatte de l'Île de Man, ainsi que la discussion à l'Académie de Médecine à Paris au début de la Grande Guerre. Aussi bien l'article « À propos de la télégonie », rédigé à partir de ladite intervention d'Anthony, et l'entrée correspondante dans le Journal de médecine et de chirurgie pratiques à l'usage des médecins praticiens en donnent témoignage et désignent l'atavisme comme l'une des explications hypothétiques de la télégonie les plus plausibles pour les spécialistes. Elle sera, par ailleurs, l'idée de base de la transmission des caractères héréditaires pour la génétique classique : le caractère dominant (qui se manifeste) ou récessif (qui ne se manifeste pas) d'un trait quelconque chez un individu. La définition fournie par le Petit Robert de la langue française (2017 : 165) pour l'atavisme présente cette possibilité de façon très synthétique : «BIOL. VIEILLI. Forme d'hérédité dans laquelle l'individu hérite de caractères ancestraux qui ne se manifestaient pas chez ses parents immédiats ; réapparition d'un caractère primitif non modifié après un nombre indéterminé de générations ». Ceci est équivalent à dire qu'un gène peut être récessif pendant plusieurs générations et récupérer le caractère dominant perdu à un point exact de la généalogie, sans que l'on doive parler pour autant d'un phénomène d'imprégnation. L'installation de l'atavisme dans le paradigme de l'hérédité n'empêche pas qu'Anthony accorde à ses collègues contemporains « un grand abus »de celui-ci. Cela fait partie du mécanisme de construction de la science à travers un processus d'essai-erreur et de cohabitation de l'approche traditionnelle et de la modernité : mythe et science. Telle est la conclusion à laquelle semble arriver le Journal de Médecine dans l'entrée consacrée à la télégonie et qui sert à résumer la discussion dans l'Académie de Médecine à Paris :

«On voit [...] combien la doctrine de la télégonie est discutable et cependant il faut reconnaître qu'elle conserve encore beaucoup d'adhérents et cela, bien qu'elle ne repose en 
somme que sur des idées qu'on pourrait appeler populaires, et plus particulièrement sur l'observation des animaux, et nullement sur des données scientifiques ». (LucasChampionnière, $1915: 463$ )

Pourquoi Zola aurait-il donc choisi une telle théorie, qui manquait de rigueur et du consensus de la communauté scientifique, comme sujet de son roman Madeleine Férat ? C'est justement le caractère hypothétique et peu rigoureux de cette doctrine qui a pu l'attirer car elle semblait bien romanesque. En ce sens, il ne serait pas loin de M. Pinard qui affirmait dans les Comptes-rendus de la Société de biologie du 2 avril 1913, repris dans le Journal de médecine et de chirurgie pratiques, que cette hypothèse de la télégonie «admise par quelques-uns appartient au roman et non pas à la biologie » (Lucas-Championnière, 1915 : 463). Par ailleurs, si l'on considère que plus d'une décennie sépare Madelaine Férat de l'essai théorique qui fixe les postulats de sa littérature - Le Roman expérimental -, on est porté à croire que Zola n'a pas vraiment pris au sérieux une théorie qui manquait d'assises scientifiquse, et qui allait entre 1868 et 1880 être questionnée par les savants. La publication de ce roman pourrait bien être le dernier échelon avant l'essor de sa grande réussite littéraire: la série de Les Rougon Macquart; une sorte de tentative expérimentale, immédiatement après abandonnée, cherchant à établir les fondements du Naturalisme et le développement de la formule magique de son œuvre littéraire, soit la synthèse entre science et littérature.

\section{L'acheminement (scientifique) vers Les Rougon Macquart : Madeleine Fé- rat}

Le parcours littéraire d'Émile Zola avant d'atteindre le succès commence en 1862, quand il est embauché chez Hachette avec à peine 22 ans. Il y connaîtra des auteurs comme Lamartine et Sainte-Beuve, ce dernier si déterminant pour son profil de critique littéraire, mais la maison d'éditions sera surtout l'école où il apprendra que la littérature est aussi affaire économique et qu'il est essentiel de frapper le public par le contenu des œuvres. Très tôt il prend conscience de la fonction sociale que peut remplir la littérature qui au dire de Cressot (1928 : 385) permet de «travailler à cette transformation des esprits et des mœurs et de réparer ainsi le mal [...] causé ». Cressot explorait justement dans cet article l'une des grandes influences de Zola pendant cette première approche aux mécanismes de l'industrie littéraire - qu'il nomme «période romantique »-, celle de Jules Michelet et sa théorie sur l'amour - De l'amour (1859) - où, parmi bien d'autres maximes, le grand historien défendait l'impossibilité de la survivance du sentiment amoureux au sein du mariage. Ces idées directrices - même si elles évolueront au courant des années et des expériences - sont déjà bien présentes dans les textes qui intègrent le «cycle de la femme » ou «romans de jeunesse » et qui supposent un apprentissage et une transition vers la consolidation de son style : La Confession de Claude, Le Vœu d'une morte, Thérèse Raquin et Madeleine Férat. Publiés entre 1865 et 1868, avec un succès inégal, ces romans présentent un scénario analogue, celui d'une femme attrapée entre deux hommes, 
l'amant et le mari, toujours dans un milieu bourgeois. Chaque roman comprend, par ailleurs, un élément significatif, assez innovateur, pour distinguer les histoires les unes des autres. C'est justement ce trait caractéristique qui retiendra notre attention car il s'avère être essentiel pour la lecture des romans qui, avec l'exception de Thérèse Raquin, ont suscité aussi peu d'intérêt chez les critiques que chez le public en général. Du reste, l'étude des textes permet d'éclairer la notion même de naturalisme en l'envisageant dans son évolution. Observer de près l'approche de la télégonie que Zola a réalisée en 1868 dans Madeleine Férat devrait permettre de voir sous un nouveau jour ses conceptions scientifiques et reconsidérer la valeur de ses premières œuvres.

Madeleine Férat est une jeune femme impulsive, passionnée et avec des traits enfantins, mariée avec Guillaume, un jeune homme timide et très peu sûr de lui-même. Malgré leurs différences, ils mènent une vie paisible dont ils ne pouvaient même pas rêver pendant une enfance qui avait été marquée par la fatalité. Élevés loin de leurs parents, tous deux ont été socialement méprisés dès leur naissance à cause des stigmates qui pesaient sur leurs géniteurs. Le mariage de convenance entre Guillaume et Madeleine suppose un oasis de repos pour les deux, seulement interrompu par les menaces de la condamnation au feu éternel que Geneviève, servante du couple et ancienne nourrice de Guillaume, prononce parfois. La raison d'être de ces mauvais augures réside dans la vie que Madeleine avait menée avant son mariage, qui faisait peser sur elle un certain manque de respectabilité. C'est que Guillaume n'était pas le premier homme de Madeleine, car auparavant, pendant presque un an, elle avait été la maîtresse de Jacques, un beau jeune homme charismatique. Le hasard, ou plutôt la fatalité, veut que toute cette vieille histoire revienne à la vie de Madeleine pour briser son calme, avec l'accouchement de Lucie, première et unique fille du couple. Sa liaison avec Jacques n'avait pas seulement affecté sa réputation, mais aussi sa propre physiologie : ayant été le premier homme à la posséder, il avait laissé en elle une empreinte perpétuelle qui se manifestait dans la ressemblance entre ses propres traits et ceux de Lucie, l'enfant que Madeleine avait eu pourtant avec Guillaume. Cependant, aucun des deux ne s'en rendent compte que jusqu'au retour de Jacques, qui s'avère être le meilleur ami d'enfance de Guillaume. Cet avènement imprévu finira par détruire l'harmonie familiale et toute tentative de mener une existence heureuse. La fatalité aura poursuivi Madeleine et Guillaume jusqu'au dernier souffle.

\subsection{Les mécanismes de l'hérédité appliqués au roman}

En 1868, la volonté de Zola d'impregner son œuvre d'un esprit scientifique était devenue de plus en plus évidente. Tout comme le lien qui l'unissait aux principes de Claude Bernard, directeur déjà à l'époque du Collège de France, qu'il admirait profondément et dont l'œuvre était pour lui une véritable source d'inspiration. Dès cette époque, il commence à tracer son histoire parallèlement à celle du savant, surtout en ce qui concerne le but de ses travaux. L'ambition de 
Zola pour Le Roman expérimental coïncide avec l'objectif principal de la science: la quête de la vérité et l'éclaircissement des phénomènes de la nature :

Notre seule besogne est d'aller toujours en avant dans la conquête du vrai, quitte à accepter les conclusions dernières. [...] La science est donc, à vrai dire, de la poésie expliquée ; le savant est un poète qui remplace les hypothèses de l'imagination par l'étude exacte des choses et des êtres (Zola, $2006: 115)$.

Science et littérature ont été bien présentes dans la vie aussi bien de Zola que dans celle de Claude Bernard, car l'arrivée de celui-ci à Paris avait été motivée par son rêve d'être écrivain. Il a vite abandonné ce projet pour commencer sa carrière scientifique, mais sa passion pour la littérature l'a accompagné pendant le reste de sa vie. C'est peut-être en l'honneur de cette vocation à jamais disparue que Zola établit un rapport si étroit la citation précédente entre le poète et le savant. Il met aussi en relief le caractère merveilleux que partagent les processus de création et de découverte de ces deux domaines. Les mécanismes intrinsèques qui dirigent leur développement gardent des mystères, dont la résolution peut être laissée en poésie au gré du lecteur, mais que la science est obligée, par sa propre nature, de dévoiler. Les travaux et le profil personnel de Zola et de Claude Bernard démontrent qu'une coupure nette entre deux disciplines apparemment si opposées n'a pas de raison d'être quand elles pourraient parfaitement représenter une source d'aide mutuelle.

La quête du vrai implique aussi bien pour l'écrivain que pour le savant l'interaction avec la nature comme moteur de recherches, mais toujours d'une façon respectueuse afin d'extraire sa propre essence et en tenant compte que, selon Claude Bernard (1865 : 159), «il faut modifier la théorie pour l'adapter à la nature, et non la nature pour l'adapter à la théorie ». Voici l'un des grands soucis de Zola pour la construction de sa théorie romanesque, le Naturalisme, dont le nom donne déjà une idée du grand enjeu du romancier : le témoignage de la vie telle qu'elle est. Il a été toujours très clair pour Zola qu'il n'était pas scientifique, mais cela ne l'a pas empêché d'adopter les formes de la méthode expérimentale, même si, de son propre aveu, «dans l'état actuel de la science de l'homme, la confusion et l'obscurité sont encore trop grandes pour qu'on se risque à la moindre synthèse». (Zola, 1880 : 60). Même si une telle synthèse dans le champ scientifique n'était pas possible à l'époque par les propres limites de la discipline, l'approche zolienne de la méthode scientifique connaît son grand succès grâce à la capacité du romancier de fusionner, tout d'abord, science et littérature et, ensuite, son profil d'écrivain et de critique.

D’un point de vue théorique, la caractérisation de l'œuvre littéraire et du texte critique est bien claire, non seulement en ce qui concerne le produit final, fictionnel et discursif-argumentatif, respectivement, mais aussi la nature de son processus de construction. En ce sens, la différence la plus remarquable réside dans le traitement que le romancier et le critique font des matériaux qui les inspirent lors de la rédaction de leurs œuvres, car de même que Darwin se battait à 
l'époque pour essayer de démontrer l'erreur de la conception de la vie par un processus de génération spontanée, il est fort difficile de penser une œuvre sans influences externes. Tandis que la production littéraire s'efforce à développer un processus de mimèsis pour effacer ou cacher les fils qui composent la toile du roman derrière les éléments de la créativité compositrice, il faut que la critique laisse explicites les sources, « ménage une large place à l'explication et à la modélisation et établisse clairement aussi les appartenances, les filiations, les dettes et les raisons d'être » (Mourad, $2007: 83$ ).

Zola est parvenu à faire une véritable synthèse de ces deux aspects tout au long de sa carrière. D'un côté, il a mis les bases d'une théorie littéraire de façon explicite dans Le Roman expérimental, comme s'il s'agissait des postulats d'un paradigme scientifique, et il les a soumis à un processus de mimèsis dans ses œuvres à travers l'emploi de découvertes scientifiques en vogue à l'époque. Si la série de Les Rougon Macquart fixe la formule magistrale du Naturalisme avec la focalisation sur des maladies et des malformations héritées, génération après génération, dans des ambiances déterminantes, Madeleine Férat ouvre la porte à la naturalisation de la science d'une façon extrême. L'ignorance qui entoure la télégonie à l'époque n'empêche pas Zola, sans pour autant entrer dans le débat de sa validité, contestation ou critique, de l'inclure dans le roman avec la douceur nécessaire pour l'œuvre et de manière vraisemblable pour le lecteur. Plus précisément, on peut dire que la télégonie devient un plasma qui imprègne le roman de la même façon que le sang coule à travers le corps humain et arrive à tous les coins de celui-ci sans qu'il soit pourtant observable du dehors. Il faut gratter la peau, se frayer un chemin entre tissus et organes - mots et lignes- pour découvrir la véritable nature du corps - textuel -. L'ensemble de l'œuvre devient ainsi une pièce à classer dans le processus de construction de l'épistémologie scientifique à l'époque, ce qui lui donne sa véritable valeur au-delà d'un simple produit littéraire (Duffy, 2009).

La haute considération accordée à l'œuvre de Zola - que l'on peut juger comme l'application radicale de la science au roman - n'est pas le produit d'un travail désordonné, erratique ou hasardeux, sinon le résultat d'un processus d'application global de la méthode expérimentale, qui commence bien avant le moment où le romancier prend la plume pour se mettre à créer. De fait, lors de la création de la première ligne du texte, Zola en avait déjà écrit des centaines suivant point par point les différentes étapes de la méthode expérimentale bernardienne, à laquelle il emprunte la plupart de ses éléments. Comme point de départ, il faut en souligner deux: l'observation et l'expérience. Bernard (1865 : 107) luimême explique clairement que la réussite du processus repose à 50\% sur chacune des deux phases du travail scientifique :

Mais l'homme ne se borne pas à voir ; il pense et veut connaître la signification des phénomènes dont l'observation lui a révélé l'existence. Pour cela il raisonne, compare les faits, les interroge, et, par les réponses qu'il en tire, les contrôle les uns par les autres. C'est ce genre de contrôle, au moyen 
du raisonnement et des faits, qui constitue, à proprement parler, l'expérience, et c'est le seul procédé que nous ayons pour nous instruire sur la nature des choses qui sont en dehors de nous. Dans le sens philosophique, l'observation montre et l'expérience instruit.

Les romans de jeunesse de Zola ne sont pas seulement un laboratoire pour l'expérimentation thématique ou stylistique, créative et compositrice. Sa volonté de faire des rues, de la campagne, bref, de la vie quotidienne, sa scène théâtrale produit une énorme quantité d'observations qu'à la manière du savant avec ses travaux de préparation, l'écrivain systématise et ordonne sous la forme de dossiers préparatoires. L'arme principale de Zola est l'enquête, qui vise l'appréhension des modes de vie, des mœurs, mais aussi de tous les détails des métiers et des occupations de ce que seront, par la suite, les héros de ses romans. Zola, dans sa volonté d'aller au-delà de l'encore très puissant réalisme, développe une méthode de travail ferme, sérieuse et rigoureuse qui lui ouvre un espace que Frédéric Giraud (2013 : 153) appelle de " légitimité littéraire ». Grâce à ce travail de terrain préalable, Zola développe et perfectionne à l'époque sa théorie des «scènes nécessaires » qui postule que les situations qui apparaissent dans ses œuvres sont celles qui lui ont semblé les plus pertinentes et vraisemblables pour chaque moment $d u$ développement du roman.

La vraisemblance chez Zola est donc étroitement liée à une situation de nécessité. Idée de base, les «scènes nécessaires » sont incompréhensibles sans une notion qui suscite des visions opposées dans le domaine de la génétique, celle du déterminisme qui, dans le cas zolien, se tourne en « déterminisme littéraire ». De cette manière, Zola dessine les personnages de telle façon qu'ils ne peuvent pas agir autrement dans des circonstances analogues. Grâce à leur impeccable élaboration, il a été parfaitement capable de connecter avec le public, ce qui était pour lui indispensable depuis le début de sa carrière. On a pu ainsi comprendre les raisons qui obligent le personnage à agir de telle façon et qu'il se reconnaisse luimême comme capable d'avoir un tel comportement dans un tel contexte. S'il s'agit de dévoiler les sources de la mimèsis de la composition, les racines de l'insistance de Zola de toucher le public à travers l'identification avec le personnage ou le contexte se trouvent clairement dans l'élucidation du moi ou la connaissance de soi-même reçues dans la théorie littéraire classique. Peut-être le lecteur ne s'en rend pas compte au début mais il y a toujours des situations dans ce genre de récit qui éveillent quelque chose qui est en lui et que l'écrivain parvient à faire sortir, un rapport net avec les mécanismes de la maïeutique socratique.

Comme si Madeleine Férat était une tentative de mener à bout tous ces postulats scientifiques dans le champ littéraire, Zola jongle avec le critère des « scènes nécessaires » en même temps qu'il les met en rapport avec le hasard pour tisser une véritable carte génétique dans le roman. Il y parle d'un choix qui répond à des critères de détermination et vraisemblance mais dont les différentes possibi- 
lités se présentent presque par hasard, obtenant ainsi un éventail de variables qui ressemble beaucoup à celui de l'hérédité humaine : déterminisme et hasard.

La structure du roman n'échappe pas à cette combinaison contradictoire qui provoque les grands bouleversements dans l'histoire et qui ont toujours à voir avec le rapport entre Jacques, Madeleine et Guillaume. Primo, Jacques se sauve miraculeusement de l'accident du bateau «Prophète » et il se rétablit après avoir été au lit plus d'une année, où il faillit mourir presque dix fois. Secondo, il retrouve Guillaume à Maintes, sans Madeleine, l'un des rares jours où il quittait la Noiraude, la maison du couple, à cause d'une affaire qui exigeait expressément sa présence. Tertio, Zola joue deux fois avec le fait que l'un des personnages rate le train pour provoquer un déclenchement inattendu des faits ou bien une rencontre imprévue. C'est, d'abord, le cas de Jacques à la gare de Maintes le jour après la confession de Madeleine à Guillaume de son passé avec Jacques, ce qui a provoqué la fuite du couple à Paris : " [Jacques] entra tout juste à Mantes pour entendre le sifflet du train qui s'éloignait » (Zola, 1999 : 257). En plus, la rencontre de Madeleine et Jacques à Paris à la fin du roman, quand elle feint d'oublier un colis et laisse aller son mari avec la promesse de prendre le train suivant, doit aussi intégrer ce groupe de coïncidences hasardeuses mais qui supposent la construction d'un réseau de situations absolument incontournables pour l'aboutissement de l'histoire. Zola parsème le roman de ces «scènes nécessaires » que l'on peut analyser à des niveaux différents. Un lecteur qui ne reste qu'en surface du texte verra bien comment la vie peut changer d'un instant à l'autre ; un lecteur plus attentif, qui remarque toute la construction scientifique du roman, trouvera dans cette structure un nouvel instant de génie de l'auteur. Pour être bien fidèles aux grands axes de la génétique classique, il nous faudrait aussi nommer la fatalité et le milieu, deux concepts intimement liés dans Madeleine Férat.

La fatalité est une idée fréquemment exploitée dans le monde de la littérature, étant donné qu'elle était déjà présente dans la mythologie grecque. Sa conception classique, celle de la fatalité comme une nécessité inévitable et qui est en quelque sorte liée au fonctionnement du monde, est personnifiée dans la figure d'Ananké, complémentaire de celle de Cronos, personnification du temps. La réception et adaptation de ce mythe de la fatalité divine tout au long de l'histoire de la littérature a été diverse et inégale. D'abord, comme un élément global adopté par le Classicisme tel qu'il était conçu par les Grecs. Par contre, les romantiques donnent une nouvelle perspective à ce mythe, très liée à la forme du récit. En fait, Victor Hugo ouvre son célèbre roman Notre-Dame de Paris en utilisant le mot « ananké » auquel il consacre tout un chapitre, où l'on peut néanmoins constater que la fatalité n'en est pas vraiment une pour les romantiques. Au bout du compte ce n'est qu'un mot inscrit sur un mur alors que le lecteur ne ressent pas la destinée de Quasimodo et Esmeralda comme une tragédie, mais plutôt comme un drame. Le sonneur de cloches est certes condamné à être séparé de la bohémienne par des forces externes, mais qui sont incarnées par d'autres personnages, par leurs passions et désirs. 
La conception de la fatalité de Zola, par contre, s'éloigne des significations divine et stylistique que l'on vient d'expliquer à cause du rapprochement qu'il fait entre cette idée et le contexte scientifique. Ceci change radicalement la perspective d'une réalité qui aura un poids spécifique dans la série de Les Rougon Macquart et dont l'acheminement commence dans Madeleine Férat. Le romancier y présente la fatalité comme un trait intrinsèque au propre individu. On peut donc ici découvrir une conception de la fatalité assez proche de la notion de destin, qui le relie directement à l'idée catholique du monde, car «l'imprégnation [dans $M a$ deleine Férat] est décrite comme une lourde fatalité. Enfermée dans les vérités de la « science », la femme n'a aucune échappatoire » (Olrik, $1986: 132)$. Les caractères de Guillaume et Madeleine étant assez pleins et agissant, ils représentent en quelque sorte, suivant le chemin prévu, la quintessence de cette "fatalité scientifique ». En ce sens, on peut parler de personnages déterminés même si, dans certains passages de l'histoire, il pourrait sembler qu'ils vont échapper à leur destin cruel, surtout Madeleine, enterrant derrière elle un passé considéré socialement honteux. Mais, au bout du compte, ce qu'ils tentaient de faire était d'aller contre leur «nature », quelque chose d'impossible aussi bien par ce que l'on pourrait envisager de sa propre génétique que par le milieu bourgeois où ils habitent, où les fautes finissent par être toujours punies. L'influence de Jules Michelet dans la construction et description de cette ambiance est à nouveau très remarquable d'autant que l'historien avait déjà analysé et trouvé les causes de l'échec du couple marié dans l'éducation et les normes sociales qui laissaient homme et femme contraints à perpétuer des valeurs et attitudes qui rendaient impossible la cohabitation entre amour et mariage. Il y a pourtant un paradoxe dans les idées de Michelet à ce propos, mais qui sert aussi à illustrer les contradictions de la considération de la nature de la femme-épouse dans Madeleine Férat, comme constate Hilde Olrik (1986 : 132) : «D'une part il [Michelet] veut la femme-nature, la vierge, l'objet de son désir. D'autre part il veut l'épouse-reflet-de-sa-propreimage. Il ne voit pas qu'en la virilisant il détruit la nature en elle ».

La puissance de cette atmosphère angoissante et de la dichotomie homme / femme-épouse prend toute sa force lors de la dernière rencontre des amants (Jacques-Madeleine), qui a lieu le jour même de la mort de la petite Lucie à cause de la vérole. Ceci dit en passant, le choix de la maladie qui a mené à la découverte du vaccin, dont les premières études ont été publiées à la fin du XVIII ${ }^{\mathrm{e}}$ siècle ne semble pas non plus hasardeux. Toujours est-il qu'ils se livrent l'un à l'autre - à strictement parler la seule infidélité de la femme envers Guillaume- à midi, le moment où Madeleine entend sonner les coups de l'horloge dans l'appartement de Jacques. Lors de son retour à la Noiraude, elle comprend la cruauté de la situation et se culpabilise elle-même :

- À quelle heure est-elle morte ? demanda-telle à Geneviève qui avait repris ses prières.

- À midi, répondit la protestante.

Cette courte réponse tomba sur la tête de Madeleine comme un coup de massue. Geneviève aurait-elle raison ? Serait-ce 
sa faute qui aurait tué sa fille ? À midi elle était dans les bras de Jacques, et à midi Lucie mourait. Cette coïncidence lui parut fatale, atroce (Zola, 1999 : 358).

Comme si l'usage du mot « fatale » était lui-même un exemple de métalittérature, la prononciation de ce mot réveille en Madeleine la constatation du fait que la fatalité était intrinsèque à elle-même et, par conséquent, qu'elle ne pouvait pas y échapper, ce qui la pousse à se suicider. Ce constat, aggravé par la situation traumatisante de la mort de sa fille, était survenu à Madeleine quelques pages auparavant, moment où l'on peut constater la naturalisation du lien entre fatalité et scientificité fait par Zola, ainsi que le rapport fort qui l'unissait à Claude Bernard comme initiateur de la physiologie :

Elle ne s'expliquait pas les fatalités physiologiques qui soustrayaient son corps à l'action de sa volonté $[\ldots]$ elle finissait par s'accuser de goûts monstrueux en voyant son impuissance à oublier son amant et à aimer son mari. [...] Elle se disait que Geneviève avait raison, qu'elle devait avoir l'enfer dans les entrailles (Zola, 1999 : 311).

L'emploi du terme « fatalité physiologique » est un bon exemple du dépassement du texte zolien comme simple artefact littéraire pour faire partie du corpus épistémologique de l'époque. L'auteur parvient à intégrer d'une façon organique des notions tirées de la science dans le roman comme si elles étaient n'importe quel autre élément de la narration. Même si des concepts comme la fatalité ou le déterminisme dépassent largement les limites de l'argument et sont ceux dont Zola se sert pour faire avancer l'histoire et pour atteindre des moments de tension dramatique qui font sortir le lecteur de la ligne monotone qui s'empare du roman, dans sa première moitié, la propre idée d'imprégnation survole à tout moment le texte, surtout à travers le mécanisme de la répétition. Son apparition est assez fréquente précisément dans ces passages plats, où l'auteur laisse des pistes au lecteur au moyen d'éléments symboliques qui se répètent constamment et qui permettent à celui-ci d'anticiper tout ce qui va se passer de façon plus ou moins fidèle. En ce sens, on peut parler d'une certaine détermination dans le roman, un autre trait commun aux œuvres de jeunesse de Zola. C'est-à-dire, il force toujours ses personnages, surtout la femme -Madeleine, dans ce cas- au moyen de sa théorie des « scènes nécessaires » à faire face à des endroits, des vêtements ou des objets que l'on peut considérer imprégnés et qui leur font penser à leurs fautes.

La présentation de l'imprégnation comme axe du roman acquiert à ce stade une nouvelle dimension. Zola pousse les postulats scientifiques observés dans le monde animal, dont l'une des grandes controverses est son manque de corrélat dans la physiologie humaine, vers le terrain de l'inanimé. Il mélange ainsi une idée qu'il emprunte à la science avec le grand débat sur le rôle que joue le milieu dans l'hérédité. Dans le cas de Madeleine Férat, l'interaction entre caractère et milieu donne des résultats manifestement déterministes. Guillaume, mais surtout Madeleine, essayent de mener la vie qu'ils sont censés avoir, en s'efforçant de 
vivre suivant les contraintes de la société bourgeoise, mais ce sont précisément les mœurs et obligations de ce milieu qui font briser la paix du couple et qui les empêchent d'échapper à leur destin. Malgré sa ferme volonté, le passé de Madeleine est une menace qui revient constamment au présent pour imprégner l'avenir et s'emparer de ses possibilités d'être heureuse. Sa vie antérieure constitue une sorte d'empreinte qui, façonnée par l'action de la mémoire et du temps, tourmente le couple et comble de remords la jeune femme. Comme s'il s'agissait d'un processus de naturalisation analogue à celui mené par Zola pour la construction du roman, on peut parler d'une imprégnation spatiale, située dans la sphère de la mélancolie et du désir et dont l'analyse faite par Annie Clément-Perrier (2007 : 28) pour Les Géorgiques de Claude Simon est parfaitement valable pour expliquer sa nature: « la maison des romans simoniens est un coffre, un écrin, un réservoir d'images et de papiers "imprégnés de ou plutôt exhalant cette odeur de renfermé, de passé prêt à resurgir"».

Claude Simon n'est pas le seul auteur à avoir eu recours à un tel mécanisme. Il est bien simple d'établir un lien entre son emploi et des écrivains qui ont influencé Émile Zola de façon bien claire. Par sa ressemblance thématique, ainsi que par la considération que le naturaliste a toujours eu envers lui, prenons Honoré de Balzac et son Chef d'œuvre inconnu (1831) comme exemple. L'atelier de maître Porbus est clairement un lieu imprégné par la mémoire d'un passé plus glorieux, mais aussi par l'influence du souvenir du modèle de sa femme idéale. De façon analogue, le fantôme de Jacques survole la vie de Madeleine dès le début du livre, quand elle est, contrainte par crainte du tonnerre à passer la nuit avec Guillaume dans la même chambre d'auberge où elle avait jadis logé avec Jacques. Sa seule présence dans la salle éveille en elle un sentiment de rage inouï :

Le lendemain, Madeleine s'éveilla la première. Elle descendit doucement du lit et s'habilla en contemplant Guillaume qui sommeillait encore. Il y avait presque de la colère dans son regard. Une indéfinissable expression de regret passait sur son front dur et grave que le sourire de ses lèvres n'adoucissait pas. Parfois, elle levait les yeux, elle allait du visage de son amant aux murs de la pièce, à certaines taches du plafond qu'elle reconnaissait. Elle se sentait seule, elle ne craignait pas de s'abandonner à ses souvenirs. À un moment, en reportant ses regards sur l'oreiller où reposait la tête de Guillaume, elle tressaillit comme si elle se fût attendue à trouver une autre tête à cette place (Zola, 1999: 50).

Zola déploie tout la force narrative de ce que l'on pourrait appeler l'« imprégnation spatiale » dans les moments les plus accablants pour les protagonistes -le constat de la ressemblance entre Jacques et Lucie, par exemple- où l'on se rend compte des modifications que le regard et l'attitude du sujet peuvent provoquer dans l'empreinte laissée par un même endroit, étant donné les règles de la mémoire et du temps qui régissent son fonctionnement. C'est le cas de l'hôtel que Madeleine loue lors de son déménagement à Véteuil, lieu de résidence de Guil- 
laume. D'abord refuge charmant des amants et symbole de sa liberté, suite au retour de Jacques, il finit par symboliser l'enfer pour le couple, un endroit diabolique et sombre :

Nous avons eu tort de venir ici ; nous aurions dû nous réfugier dans quelque chambre inconnue où nous n'aurions pas trouvé, vivant et cruel, le souvenir de nos anciennes amours. Si cette couche où nous avons dormi, si ces fauteuils où nous nous sommes assis, ne nous paraissent plus avoir les tiédeurs de jadis, c'est que nos corps eux-mêmes les glacent. Tout est mort en nous (Zola, 1999: 216).

Même si l'on a remarqué que l'imprégnation spatiale se développe dans le domaine de l'inanimé, Zola se place à un certain passage dans le seuil entre celuici et les corps vivants. À un moment donné, il semble que dans le va-et-vient, les corps de Madeleine et de Jacques subissent une symbiose avec le propre espace. Rapprocher la figure de la femme et celle d'un bâtiment ou d'une chambre, même si cela peut paraître frivole, est une question plus commune qu'il ne semble. Guy de Maupassant en donne la preuve dans son récit Une ruse : "Rien de plus vrai. Une femme ne peut aimer passionnément qu'après avoir été mariée. Si je la pouvais comparer à une maison, je dirais qu'elle n'est habitable que lorsqu'un mari a essuyé les plâtres. »(1891: 365). L'expression «essuyer les plâtres », utilisée lorsqu'un immeuble est habité pour la première fois, précise le rapport étroit entre la femme et un bâtiment et contribue à la réification de celle-ci comme élément accessoire. Car, de même que le plâtre n'est qu'un matériel ornemental, inutile pour la structure du bâtiment, la femme bourgeoise apparaît ici comme une matière que l'on moule, que l'homme moule par son action généreuse.

\subsection{Le rapport homme-femme dans la télégonie.}

Outre les aspects scientifiques de l'imprégnation que Zola accorde à $M a$ deleine Férat, que l'on aborde l'explication de cette théorie depuis la perspective de la femme, de l'homme ou de leur descendance, on gravite toujours autour d'une même idée: l'inachèvement. La femme est un être incomplet qui doit être perfectionné par l'homme au moyen des rapports sexuels. Quelle que soit la métaphore que l'on utilise pour faire allusion à cette représentation de la femme, on finit par retrouver le mythe d'Adam et Ève.

Nul hasard à ce que ce récit, le deuxième du livre de la Genèse, soit placé immédiatement après l'explication de la création du monde, véritable louange aux merveilles de Dieu. Il en ressort une humanité qui, contrairement à son créateur, se montre ingrate et incapable de respecter la seule condition qu'on lui impose pour demeurer au Paradis: ne pas manger de l'arbre de la connaissance du bien et du mal. Ève étant responsable par son infraction de l'expulsion du couple qui condamne l'humanité à l'exil du Jardin de l'Eden, la femme restera à jamais figée dans cette image de pécheresse. Or, Zola exploite largement cette image dans son roman à travers la menace que suppose pour Madeleine sa vie antérieure au mariage, ce qui suppose une nouveauté par rapport à l'approche de Michelet à la di- 
chotomie amour-mariage. Zola introduit des touches d'érotisme et de sensualisme dans l'attitude de Madeleine qui n'étaient pas prises en compte par l'historien (Cressot, 1928). Mais ce qui nous intéresse davantage pour finir de comprendre la naturalisation de l'imprégnation que Zola développe dans le roman, ce sont les conditions de la création divine de l'être humain une fois le monde achevé en six jours. Le livre de la Genèse explique clairement comment Dieu a créé d'abord l'homme « à Son image », à partir de la poussière, et ensuite la femme, pour donner au mâle un être semblable après avoir créé les bêtes auparavant. C'est ainsi que «L'Éternel Dieu forma une femme de la côte qu'il avait prise de l'homme, et il l'amena vers l'homme». Adam l'a tout de suite reconnue comme « os de mes os et chair de ma chair! On l'appellera femme, parce qu'elle a été prise de l'homme ». Les liens sont dès lors fixés: Dieu créé l'homme d'où surgit la femme. Celle-ci a donc une double dette : d'une part avec la divinité à laquelle elle doit son existence, mais aussi avec l'homme par l'intermédiaire duquel il l'a créée.

Or, dans Madeleine Férat Zola semble consolider la télégonie au moyen de ce mythe fondateur. Voilà peut-être pourquoi il est aisé de relever des ressemblances entre la terminologie employée dans la description du processus de l'imprégnation subi par Madeleine et le récit de la création:

Lorsque Madeleine s'était oubliée dans les bras de Jacques, sa chair vierge avait pris l'empreinte ineffaçable du jeune homme. Il y eut alors mariage intime, indestructible. [...] son corps puissant, son tempérament mesuré se laissa pénétrer d'autant plus profondément qu'il était riche de sang et sain d'humeurs. [...] On eût dit que Jacques, en la serrant contre sa poitrine, la moulait à son image, lui donnait de ses muscles et de ses os, la faisait sienne pour la vie. Un hasard l'avait jetée à cet homme, un hasard la retenait dans son étreinte, et, pendant qu'elle était là, par aventure, toujours sur le point de devenir veuve, des fatalités physiologiques la liaient étroitement à lui, l'emplissaient de lui. Lorsque, après une année de ce travail secret du sang et des nerfs, le chirurgien s'éloigna, il laissa la jeune femme éternellement frappée à la marque de ses baisers, possédée à ce point qu'elle n'était plus seule maîtresse de son corps; elle avait en elle un autre être, des éléments virils qui la complétaient et l'asseyaient dans sa force. C'était là un phénomène purement physique (Zola, 1999 : 231).

Ce passage contient non seulement les traits principaux de la notion de « femme imprégnée », mais aussi la trace d'une cartographie exhaustive de la naturalisation zolienne des savoirs scientifiques qui intègrent le roman, implicitement ou explicitement. En ce sens, l'image la plus éclaircissante est peut-être celle d'une divinité devenue chirurgien dans le contexte du XIX ${ }^{\mathrm{e}}$ siècle, qui prend de la distance pour contempler son travail ; on dirait même un peintre qui admire son chef-d'œuvre. Lui, qui a moulé la femme à son image, grâce à la médiation incontournable d'éléments qui se trouvaient au centre de l'éclatement des disciplines 
scientifiques à l'époque - sang et nerfs -. L'expression «travail secret » décèle à nouveau la place instable de la science à l'époque, à cheval entre le connu et l'inconnu. Par ailleurs, la métaphore de la côte est dans ce cas menée à l'extrême pour montrer comment la femme est présentée comme un être ayant besoin de l'homme même pour se tenir debout et marcher. Étant donné que les muscles et les os sont les parties clés de la posture bipède, son absence condamnerait la femme à une vie semblable à celle d'une plante. Sur ce point on constate une autre nouveauté en ce qui concerne l'évolution chez Zola des idées empruntées à Michelet, dont on a fait allusion auparavant, et qui constituent un tournant décisif vers le dénouement tragique de l'histoire : «La métamorphose de la femme en homme, qui était, chez Michelet, une preuve de l'union sacrée entre le mari et sa femme, est devenue une force destructive »(Olrik, 1986 : 134).

Le plan de naturalisation de Zola repose sur une double mythologie : la chrétienne, déjà mentionnée, et la classique. On peut considérer que la soi-disant scientificité de la télégonie est renforcée par un autre mythe. En effet, le rapport entre cette approche à la figure de la femme et celle du mythe de Pygmalion est assez évident. Le processus narré par Ovide dans Les métamorphoses insiste encore sur le fait de présenter la femme comme un moule, comme un matériel qui a peu de valeur jusqu'à ce qu'il est frappé, restant toujours sur le plan physique de la question, tandis que Zola va un peu plus loin en faisant attention aussi à la dimension sociale ou psychologique de la question. Même si d'après le récit classique la statue était faite en ivoire, un matériel très valorisé, ni celle-ci ni Madeleine n'étaient complètes avant de connaître leurs maîtres, Pygmalion et Jacques, respectivement. Elles étaient de belles potiches qui n'avaient atteint leur plénitude qu'après avoir été imprégnées par un homme. En ce sens, il ne semble pas que le verbe « frapper» ait été choisi par hasard, sinon par ses implications dans le processus de formation des monnaies, où l'on constate un parallélisme assez illustratif avec l'imprégnation. Un matériel simple, tel que le métal, acquiert une valeur déterminée et largement supérieure à l'initiale une fois qu'il subit un simple processus mécanique, une fois qu'il est frappé.

De même qu'il existe plusieurs explications théoriques, non validées, sur la télégonie, l'analyse du rôle de l'homme, responsable de la transformation de fille en femme, est donc incontournable pour ces idées, et peut être également considéré sous plusieurs perspectives. Le portrait que Zola fait des personnages de Jacques et de Guillaume confronte le lecteur aux positions les plus extrêmes que l'on puisse considérer. Jacques est présenté comme un être puissant, maître absolu du rapport avec Madeleine, non seulement par l'empreinte qu'il laisse en elle en tant que mâle, mais aussi car il est le responsable de son entrée dans les cercles bourgeois et de son enrichissement intellectuel. Zola essaie de transférer la force et l'importance de ce lien au moyen du vocabulaire employé («chair vierge » et «empreinte ineffaçable », c'est-à-dire, liés pour toujours). À côté de ce rôle de « tuteur », l'amant est le propriétaire et protecteur de la femme (« la faisait sienne pour la vie »). Ce sentiment de possession, l'une des bases de la théorie de 
l'imprégnation, sera présent non seulement pendant le temps où Madeleine et Jacques ont une liaison, mais pour toujours. Même si cette idée est partagée aussi bien par Jacques que par Guillaume, les deux hommes de la vie de Madeleine, ils ne sauraient être plus différents. Le fait que la petite Lucie ressemble à Jacques mais soit la fille légitime de Guillaume doit s'interpréter non seulement par rapport à la figure de Madeleine, mais aussi à celle de Guillaume et d'un soi-disant «manque de masculinité » latent dès le début de l'œuvre.

Guillaume est présenté comme peu capable de féconder une femme : «fils intelligent et affaibli d'une forte race », «d'une timidité d'enfant », «d'une sensibilité de femme » (Zola, 1999 : 34). Zola s'efforce d'accentuer cette image peu virile de Guillaume, d'autant plus que sa description est faite juste après celle de Madeleine, à cheval entre «une dureté presque masculine » et une «délicatesse exquise » dans son visage (Zola, 1999 : 33). Par conséquent, on assiste à une sorte de renversement des rôles traditionnels dans le couple : elle représenterait la vigueur et la puissance «masculine » face à la personnalité faible, malheureuse et morne de son mari.

Ce rapport asymétrique entre les mariés est analogue à celui que Guillaume entretient avec Jacques dès le début de son amitié à l'école, et qui éclatera définitivement à l'âge adulte. À un moment donné, lors du retour de Jacques à Véteuil, celui-ci condense cette différence de caractère en ayant recours à un parallélisme bien scientifique: "Comprends donc que nous n'avons pas le même sang dans les veines » (Zola, 1999 : 185), à image du «travail secret du sang et des nerfs » auquel Zola avait déjà eu recours pour faire référence aux mécanismes de l'imprégnation.

Une autre conséquence qui découle directement de la notion de « femme imprégnée » est, comme défend Anne Carol (2006 :7), en citant Boissard, un accoucheur, « une forte valorisation de la virginité, à laquelle est conférée une signification très forte ; c'est «l'ensemencement du terrain vierge qui donne le caractère ineffaçable, en assurant la fixité et la permanence ». Le rôle central de la virginité dans la sexualité féminine dans le monde occidental est directement issu de la tradition chrétienne comme réinterprétation et adaptation du mythe d'Adam et Ève du péché originel. Dans le cas de Madeleine Férat, on y trouve d'ailleurs la « lourde fatalité » de laquelle Hilde Olrik parle dans son article. Pourtant, dans la citation antérieure, la virginité est étroitement liée à la conception de la maternité suivant les postulats de la mythologie classique, qui établie un lien entre la maternité et la fertilité avec la terre. Ce rapport est incarné par Gaïa, «Déesse-mère » dans la mythologie grecque dont le nom signifie «terre» et qui existe dans presque toutes les grandes civilisations. En outre, l'identification de la femme avec un «terrain vierge » insiste sur sa réification et implique sa réduction à un rôle passif d'acheminement vers la maternité, presque sa seule fonction au sein de la société bourgeoise, dont les normes constituent, à nouveau, une série de contraintes opprimantes telles que Michelet les conçoit. 


\subsection{La dimension sociale de l'hérédité dans Madeleine Férat}

Il n'est pas étonnant que le titre original de ce roman lors de sa publication en feuilleton ait été La honte, car ce sentiment est le grand dominateur de toute l'œuvre, très liè à la notion de télégonie et son vaste domaine d'influence. Il s'agit en fait du nœud thématique de Madeleine Férat, roman qui soulève bien des questions morales délicates, car, comme le dit Zola dans l'Avertissement, il y a «au fond des cœurs, beaucoup de laides choses ». Il se penche à nouveau sur le double versant

- scientifique et social - qu'il exploitera constamment dans la série des « Rougon Macquart ». D'un côté, le cœur comme moteur de l'organisme et, de l'autre, comme image de nos passions les plus acharnées et nos secrets les moins avouables, ceux que Zola met devant le lecteur dans les romans. Il défend, dans ce même Avertissement et suivant le double caractèr de son œuvre, la convenance de la lecture d'un tel genre de roman pour faire avancer une société, suivant en cela « le dévouement du médecin qui passe sa vie dans l'étude des misères du corps, pour guérir et pour élargir la science» (Zola, 1999 : 28).

Si l'on considère Madeleine Férat comme une œuvre où l'on démontre que les arguments scientifiques sont valables pour présenter ouvertement les faiblesses et l'hypocrisie des mœurs de la société bourgeoise, combinées avec les postulats du dogme catholique, ce qui suppose la stigmatisation de la femme, élément où repose la composante socioreligieuse du roman, il n'est pas du tout étonnant que le procureur général ait menacé avec un procès de censure préventive avant même sa publication en volume. L'écrivain se voit alors obligé à rédiger un avertissement à son œuvre pour refuser l'enlèvement d'un certain passage car « les quelques lignes qu'on voudrait me faire enlever contiennent toute la thèse du livre » (Zola, $1999: 23)$. Ceci lui permettra de fonder sa défense sur des arguments scientifiques et sur des auteurs si réputés que Michelet ou le docteur Lucas, contrairement à la tendance à l'époque de recourir à des argumentations morales. La naturalisation est à nouveau sa grande arme secrète, car il se retranche nettement derrière des postulats scientifiques qu'il insère dans la vie quotidienne pour faire une critique sociale voilée. Zola trouve dans la télégonie un tel prétexte, ainsi que le squelette qui supporte les misères humaines, matériel honteux qu'il faut cacher et qui constitue le muscle de l'œuvre. Ainsi le prouve le fait que Madeleine ne parle jamais de Jacques à son mari et que celui-ci n'ose pas l'interroger sur sa vie antérieure. La vérité est latente mais mari et épouse font la sourde oreille. En fait, Guillaume et Madeleine symbolisent une sorte d'oasis au milieu du désert pour l'autre, une bouffée d'air frais dans son existence pénible, marquée par l'incontournable jugement social dès leur enfance. Bref, un mariage de convenance qu'ils reconnaissent comme tel et qui a permis à Madeleine de «nettoyer » son passé : «Quand tu m'as donné ton nom, il m'a semblé que tu me lavais de toute souillure » (Zola, $1999: 193)$.

On arrive ainsi à une nouvelle duplicité dans la présentation de la télégonie dans le roman. Si l'une des hypothèses expliquant l'imprégnation parle d'un pre- 
mier rapport sexuel et d'un coït ultérieur qui donnerait le dernier élan pour la reproduction, dans le cas de Madeleine le rapport sexuel de Jacques aurait fait d'elle, une vierge, une femme, tandis que le mariage avec Guillaume - et non pas ses relations intimes - l'a transformé en Madame de Viargue, nom qui la distingue et lui confère une identité au sein de la société. Il est pourtant étonnant et significatif que ce patronyme ne soit jamais employé dans le roman pour la désigner, mais bien son nom de célibataire, Férat, celui de son père, qui donne son titre au livre. Ceci peut être dû au déterminisme de la personnalité ferme de Madeleine, que l'on expliquera tout de suite, elle qui n'a jamais su s'adapter à la réalité des mœurs bourgeoises.

Le chemin n'avait pas été facile, et non seulement au cours de sa vie antérieure, car elle était stigmatisée depuis sa naissance. Madeleine est «née d'une morte » (Zola, 1999 : 55), ce qui marquera profondément les deux premières années de sa courte vie, pendant lesquelles son père affirme qu' "elle est frêle et délicate comme sa mère [...] elle mourra comme elle »(Zola, 1999 : 55). Bien que très faible et malade, les trajectoires de mère et fille ne pourront pas être néanmoins plus divergentes. «Elle [Marguerite] avait une de ces figures douces et soumises qui touchent les hommes forts » (Zola, $1999: 52$ ), tels que son mari, le rude Férat, qui avait même cru retrouver dans sa femme l'image d'une Vierge dont sa mère lui parlait quand il était petit.

Il est évident que la personnalité de Madeleine est tout à fait différente et, par ailleurs, elle a su s'éloigner d'un tel fantôme. Son père en est en grande mesure le responsable, l'ayant élevée avec une personnalité forte, « un esprit net, la décision rapide et logique de sa nature d'ouvrier » (Zola, 1999 : 59). Son père, audelà de sa personnalité morne et solitaire, accentuée par les tragiques circonstances de la mort de sa femme, connaissait l'importance des conventions sociales. C'est pourquoi il s'est beaucoup soucié d'épargner de l'argent pour l'avenir de Madeleine, ce qui a permis d'abord de l'élever dans un pensionnat, où l'on enseignait à maîtriser des règles qui dominaient le panorama social du $\mathrm{XIX}^{\mathrm{e}}$ siècle, pour ensuite, une fois sa formation achevée, disposer de rentes suffisantes pour mener une vie confortable. Tel était également le souci de $\mathrm{M}$. de Viargue, père de Guillaume, qui ayant aussi souffert les rigueurs de l'indifférence sociale, ne voulait pas le même destin pour son fils. Voici une troisième dimension de l'hérédité : la dimension économique.

Le manque de vigueur et sa personnalité morne n'est pas le seul stigmate qui poursuit Guillaume dès sa naissance. De même que sa maîtresse, il subissait les blagues et le mépris de gens à cause des fautes de ses parents. Sa mère, ayant laissé son mari, notaire de Véteuil, pour vivre avec $M$. de Viargue, le père de Guillaume, abandonnera sa nouvelle famille deux années après pour reprendre sa vie antérieure. D'après le narrateur, ceci répond à une raison très simple : " elle obéissait à son sang bourgeois, elle s'était faite dévote et prude » (Zola, 1999 : 76). Sa condition sociale n'a pas aidé à effacer dans un premier moment un scandale dont tout le monde parlerait bien d'années plus tard, mais lui a permis d'être 
acceptée à nouveau du bras de son mari dans les réunions les plus distinguées. Sa vie étant réglée après son aventure avec $M$. de Viargue, la cible des médisances a été son fils, le petit Guillaume, surnommé pour toujours « le bâtard ». Les abus de ses camarades à l'école ne faisaient que compléter l'indifférence de son père et les jugements implacables de Geneviève, sa nourrice et représentante des mœurs catholiques dans le roman. Son attitude envers Guillaume se balançait entre la figure maternelle qu'elle a dû être pour le petit après la fuite de sa mère et une ferme gardienne morale qui lui rappelait à chaque instant qu'il était « un fils du péché, tu expies la faute des coupables » (Zola, 1999: 85). D’après Geneviève, la vie de M. de Viargue était aussi susceptible que celle de son ancienne maîtresse d'être emparée par le péché : celui-ci était scientifique, ce qui venait couronner une vie peu attachée aux mœurs de l'époque.

M. de Viargue était né en Allemagne après la fuite de ses parents, riches propriétaires, pendant la Révolution Française et, après la mort violente de ceuxci, il n'avait jamais pardonné la France, où il se sentait comme un exilé, considérant ses habitants comme des traitres. En plus, il n'a jamais suivi les contraintes bourgeoises, ce qui a provoqué que « les habitants de Véteuil nourrissaient contre M. de Viargue une haine sourde, faite de jalousie et de pruderie ; ils ne lui pardonnaient pas d'être riche et d'agir à sa guise » (Zola, 1999: 83). Cette solitude et cet isolément n'inquiétaient guère $M$. de Viargue, même quand son fils souffrait les rigueurs morales de la petite société de Véteuil, une même façon d'agir qu'il adoptait dans ses recherches scientifiques. Car il s'écarte de l'image du savant qui partage ses efforts et ses résultats avec d'autres chercheurs pour créer ainsi un réseau d'aide scientifique réciproque. $M$. de Viargue fait ses travaux seul, dans un laboratoire où personne ne peut entrer, même son fils, qui y pénètre pour la première fois lors de la mort de son père.

Cette pièce est encore un exemple de « lieu imprégné », car d'après Geneviève, là « il [M. de Viargue] activait de son haleine le feu de l'enfer » car «elle le crut fermement devenu sorcier » (Zola, 1999 : 77). Cette affirmation met à nouveau le lecteur dans l'horizon de rupture qui suppose le développement de la science à l'époque de la publication du roman. Les idées de Geneviève sur le métier scientifique s'enracinent dans un mythe bien ancré dans l'imaginaire social depuis longtemps, le rapport entre la science et la sorcellerie, largement diffusé par la religion catholique. Malgré sa vie solitaire, M. de Viargue était conscient que son fils ne devait pas mener une existence comme la sienne, notamment à cause de la mauvaise réputation sociale que ceci entraînait. Lors d'une discussion à propos de son avenir, il demande à son fils:

- Surtout, dit-il, jurez-moi que vous ne vous occuperez jamais de science. Après ma mort, vous fermerez cette porte et ne l'ouvrirez plus. C'est assez qu'un de Viargue se soit oublié ici pendant une existence entière... Je compte sur votre parole, monsieur : vous ne ferez rien et vous tâcherez d'être heureux (Zola, 1999 : 91).

Puis il lui confirme son seul vœu pour lui : 
- Vous serez riche, continua-t-il d'un ton légèrement amer, vous pourrez être un sot et un heureux homme, si vous avez la chance de comprendre la vie. Je regrette déjà de vous avoir fait donner quelque instruction. Chassez, mangez, dormez, tels sont mes ordres. Cependant, si vous aviez du goût pour la culture, je vous permettrais de piocher la terre (Zola, 1999: 90).

Aussi bien Guillaume que Madeleine sont donc contraints à osciller toujours dans la faible ligne qui divise l'indépendance et la répétition des erreurs de leurs parents. Les parallélismes au niveau social entre Guillaume et Madeleine sont évidents, mais accentués par Zola en utilisant la même structure pour expliquer l'origine de la stigmatisation chez l'un et l'autre. Le lecteur se rend ainsi compte du souci des parents, d'autant plus qu'ils montrent des traits dès leur enfance qui pourraient les acheminer vers un destin pénible. Pourtant, malgré les efforts, il semble que le déterminisme héréditaire a au bout du compte joué son rôle, car les protagonistes ont une existence que l'on pourrait qualifier globalement de malheureuse, à l'exception de quelques mirages paisibles. Analysant ceci du point de vue de la psychologie des caractères en diachronie, il y a un manque net d'évolution des personnages, au-delà de l'influence du milieu sur leur personnalité. Zola commence dans ce roman à établir des bases qu'il développera largement dans Les Rougon Macquart, ce que l'on pourrait appeler une « hérédité télique ». Les personnages sont essentiellement déterminés à suivre un parcours et à agir d'une façon précise -ceci aide évidemment l'approche des «scènes nécessaires $\gg-$ mais des facteurs externes hasardeux peuvent toujours les troubler.

\section{Conclusions}

Les sommets atteints tout au long du $\mathrm{XX}^{\mathrm{e}}$ siècle par les sciences naturelles ne peuvent pas être compris sans la figure de Claude Bernard et d'autres savants du XIX ${ }^{\mathrm{e}}$. Ces travaux, qui sont à la base de toutes les connaissances postérieures, s'emparent encore beaucoup du mythe, un élément incontournable pour comprendre la réalité actuelle de la science et dont les scientifiques veulent à tout pris s'éloigner, en oubliant qu'il y a longtemps mythe et science entretenaient des liaisons bien plus étroites de ce que l'on est enclin à imaginer. C'est pourquoi il n'y a pas lieu de sous-estimer l'approche des théories reproductives présentée par la télégonie, non seulement parce qu'elle constitue une partie importante de l'histoire de l'hérédité, mais aussi parce qu'elle a eu ses appuis à l'époque.

De la même façon que la science s'est éloignée peu à peu du mythe, Les Rougon Macquart supposent pour Zola l'achèvement de son projet le plus ambitieux. Cette immense série supposerait l'essor de son œuvre littéraire, par rapport à laquelle Madeleine Férat se trouverait encore à un stade mythique, de même que le reste de ses romans de jeunesse. Le travail du romancier représenterait dès lors un véritable processus d'essai et erreur. On ne doit pas cependant mépriser le dernier de ses romans de jeunesse comme sujet d'analyse d'autant plus qu'il y réussit l'application de la méthode expérimentale dans sa dimension sociale. Elle est par- 
faitement constatable à tous les niveaux d'analyse et aussi de l'écriture, qui ne peut pas être ternie par les préjudices scientifiques de manque de rigueur dont la télégonie est l'objet. L'écrivain même semble être conscient de la maturité acquise par le modèle du «roman expérimental » ou « roman naturaliste » qu'il développera dans Les Rougon Macquart et Madeleine Férat est, dans la pratique, le seuil d'entrée à cet univers. Il y déploie les idées clés de son projet littéraire : déterminisme, fatalité, ambiance et hasard.

Sans doute, le trait le plus remarquable de ce roman quant à ce parcours est cette naturalisation qui dépasse toutes les objections envers la télégonie que les lacunes dans la science n'étaient pas capables de remplir. Un facteur clé dans ce processus est la pluralité de perspectives que Zola utilise pour aborder la notion d'hérédité et les rapports entre les dimensions scientifique, économique et sociale. Le romancier réussit à tisser une toile entre les trois domaines. Quant aux postulats purement scientifiques, ils restent dans un second plan, comme seule excuse pour faire une dissection minutieuse de la société bourgeoise, de ses mœurs et surtout de ses hontes, celles qui doivent se cacher dans une réalité où les apparences règnent et où rien ne peut se trouver au-dessus des conventions sociales. En quelque sorte, pour un lecteur contemporain, Zola constitue un nouveau chapitre de la mythification du modèle bourgeois à travers la propre naturalisation de ses misères, déjà trop stéréotypé de nos jours par l'importance et le symbolisme qu'il a eu du point de vue sociologique pour l'histoire de l'humanité.

\section{RÉFÉRENCES BIBLIOGRAPHIQUES}

ANTHONY, Raoul (1900): «À propos de la télégonie». Bulletins de la Société d'anthropologie de Paris, $\mathrm{V}^{\mathrm{e}}$ série, 1, 18-35. URL : https://www.persee.fr/doc/bmsap_0301-8644_1900_num_1_1_7577.

BERNARD, Claude (1872) : De la physiologie générale. Paris, Librairie Hachette et Cie.

BERNARD, Claude (2008) : Introduction à l'étude de la médecine expérimentale. Paris, Le livre de Poche («Les classiques de la philosophie»).

CARLSON, Marvin (1985) : «Ibsen, Strindberg, and Telegony ». PMLA, 100:5, 774-782. URL : https://doi.org/10.2307/462097.

CAROL, Anne (2006) : «La télégonie, ou les nuances de l'hérédité féminine ». Rives nord-méditerranéennes, $24, \quad 11-21 \quad$ URL : http://journals.openedition.org/rives/550.

CLÉMENT-PERRIER, Annie (2007) : «L'empreinte et ses pouvoirs chez Claude Simon ». Littérature, 147:3, , 21-37 URL : https://www.cairn.info/revue-litterature-2007-3page-21.htm.

CRESSOT, Marcel. (1928) : «Zola et Michelet. Essai sur la genèse de deux romans de jeunesse: La Confession de Claude, Madeleine Férat ». Revue d'histoire littéraire de la France, 35:3, 382-389. URL : https://www.jstor.org/stable/40518979. 
DUFFY, Larry (2009) : «Incorporations hypodermiques et épistémologiques chez Zola. Science et littérature ». Revue romane, 44:2, 293-312.

GIRAUD, Frédérique (2013) : «Quand Zola mène l'enquête : le terrain comme caution scientifique ». Ethnologie française, 43:1, 147-153. URL : https://www.cairn.info/revue-ethnologie-francaise-2013-1-page-147.htm.

LUCAS-CHAMPIONNIÈRE, Paul (1915) : Journal de médecine et de chirurgie pratiques à l'usage des médecins praticiens. Paris, $\mathrm{Au}$ bureau du Journal. URL: https://gallica.bnf.fr/ark:/12148/cb34348793z/date1915.

MAUPASSANT, Guy (2014 [1891]) : Contes et nouvelles (édition augmentée). Paris, Arvensa éditions.

MOURAD, François-Marie (2007) : «Zola critique littéraire, entre Sainte-Beuve et Taine ». Revue d'histoire littéraire de la France, 107:1, 83-103 URL: https://www.cairn.info/revue-d-histoire-litteraire-de-la-france-2007-1-page83.htm.

OLRIK, Hilde (1986) : «La théorie de l'imprégnation ». Nineteenth-Century French Studies 15:1/2, 128-140 URL : https://www.jstor.org/stable/23532424?seq=1.

REY-DeBOve, Josette \& Alain REY, [dir.] (2017) : Le Petit Robert de la langue française. Paris, Le Robert.

SÁNCHEZ Ron, José Manuel (2001) : El jardín de Newton. La ciencia a través de su historia. Barcelone, Crítica.

STERNE, Laurence (1841 [1759] ) : Vie et opinions de Tristram Shandy, gentilhomme. Traduction de M. Léon de Wally. Paris, Bibliothèque Charpentier.

VARIGNY, Henry de (1897) : «La télégonie ». Journal des débats politiques et littéraire, 250, 9 septembre. URL : https://gallica.bnf.fr/ark:/12148/bpt6k4688681.item.

WilgauX, Jérôme (2006) : "Corps en parenté en Grèce ancienne », in F. Prost. \& J. Wilgaux (dirs.) : Penser et représenter le corps dans l'Antiquité. Rennes, Presses Universitaires de Rennes.

ZOLA, Émile (2006 [1880]) : Le Roman expérimental. Paris, Éditions Flammarion.

ZolA, Émile (1868) : Madeleine Férat. Paris, Éditions A. Lacroix et Cie.

ZolA, Émile (1976) : Madeleine Férat. Paris, Le Livre de Poche.

ZolA, Émile (1999): Madeleine Férat. Édition et préface de Henri Mitterand, Paris, Mémoire du livre.

ZolA, Émile (2020) : Madeleine Férat. Préface de Gérald Ducheim. París, Le Chat Rouge. 